\title{
Geometric aspects of transversal Killing spinors on Riemannian flows
}

\author{
Nicolas Ginoux • Georges Habib
}

Received: 19 February 2008 / Published online: 1 August 2008

(C) Mathematisches Seminar der Universität Hamburg and Springer 2008

\begin{abstract}
We study a Killing spinor type equation on spin Riemannian flows. We prove integrability conditions and partially classify those flows carrying non-trivial solutions.
\end{abstract}

Keywords Foliations $\cdot$ Spin geometry

Mathematics Subject Classification (2000) 53C12 · 53C27

\section{Introduction}

Killing spinors on Riemannian spin manifolds are smooth sections of the spinor bundle of which covariant derivative is proportional to the Clifford multiplication. Those manifolds carrying non-zero Killing spinors have been well understood for a long time. Such a manifold is Einstein; if it is furthermore compact and non Ricci flat, then it cannot carry any non-zero non-trivial parallel form (see e.g. [16]). In particular, the existence of non-zero Killing spinors imposes very rigid conditions to the geometry of the underlying manifold.

In this article we transpose the Killing spinor equation to the set up of Riemannian flows [11], which roughly speaking, are local Riemannian submersions with 1-dimensional fibres (see Sect. 2 for the definition). These contain among others all $\mathbb{S}^{1}$-bundles with totally geodesic fibres, all manifolds carrying a non-zero parallel form and all Sasakian manifolds [10]. In the definition of that equation - that we call transversal Killing spinor-we allow the first

Communicated by V. Cortés.

N. Ginoux $(\bowtie)$

Institut für Mathematik-Geometrie, Universität Potsdam, Am Neuen Palais 10, 14469 Potsdam, Germany

e-mail: ginoux@math.uni-potsdam.de

G. Habib

Max-Planck Institut für Mathematik in den Naturwissenschaften, Inselstrasse 22, 04103 Leipzig,

Germany

e-mail: habib@mis.mpg.de 
derivatives of the spinor field to behave differently along the leaves and along the orthogonal distribution of the foliation respectively, see Definition 3.1. There are several motivations for this study. Historically those spinor fields first appear in the limiting case of an eigenvalue estimate proved by B. Alexandrov, G. Grantcharov and S. Ivanov for the Dirac operator on compact Riemannian spin manifolds with a parallel 1-form [1, (8)]. On the other hand, they stand for the most natural tools in the study of the spectrum of the Dirac operator on submersions over real space forms $[14,15]$.

The paper is organized as follows. First we recall basic facts on spin Riemannian flows and we prove integrability conditions for flows admitting non-zero transversal Killing spinors, see Theorem 3.4. It should be noticed that the resulting geometric conditions hold up to homothetic deformations of the metric along the leaves (see Lemma 3.3). We then describe in Proposition 3.6 important examples of Riemannian flows carrying transversal Killing spinors, which arise as submersions over manifolds with Killing spinors. In Sect. 4, we translate the results of Theorem 3.4 into the Sasakian setting. This imposes geometric conditions on the manifold, such as $\eta$-Einstein (see Proposition 4.1). Moreover, we show that transversal Killing spinors can be related to classical ones, see Proposition 4.3. As an example, we describe all transversal Killing spinors on the Berger spheres (here beware of our definition of Berger spheres, see Sect. 4.3). In the last section, we restrict ourselves to 3dimensional Riemannian flows and we end with the classification of compact 3-dimensional $\eta$-Einstein minimal Riemannian flows carrying non-zero transversal Killing spinors.

\section{Preliminaries}

Throughout the paper the triple $\left(M^{n+1}, g, \mathcal{F}\right)$ will denote an $(n+1)$-dimensional Riemannian manifold endowed with a Riemannian flow $\mathcal{F}$ given by a smooth unit vector field $\xi$. That means, the integral curves of $\xi$ are the leaves of $\mathcal{F}$ and for all $Z, W$ orthogonal to $\xi$ the relation holds $\left(\mathcal{L}_{\xi} g\right)(Z, W)=0[11,22]$ where $\mathcal{L}_{\xi}$ is the Lie derivative in the direction of $\xi$. Recall from this hypothesis, the endomorphism field $h:=\nabla^{M} \xi$ (known as the O'Neill tensor [20]) of the normal bundle $Q=\xi^{\perp}$ is skew-symmetric w.r.t. the induced metric $g$. Hence, one may associate a 2 -form $\Omega$ to $h$ on $Q$ through $\Omega(Z, W):=g(h(Z), W)$ for all sections $Z, W \in \Gamma(Q)$.

Moreover, the normal bundle $Q \rightarrow M$ carries a natural covariant derivative $\nabla$ defined for every section $Z$ of $Q$ by [24]

$$
\nabla_{X} Z:= \begin{cases}{[\xi, Z]^{Q}} & \text { if } X=\xi, \\ \left(\nabla_{X}^{M} Z\right)^{Q} & \text { if } X \in \Gamma(Q),\end{cases}
$$

where $\nabla^{M}$ denotes the Levi-Civita connection on $T M$ and $(\cdot)^{Q}$ the orthogonal projection onto $Q \subset T M$. From its definition the covariant derivative $\nabla$ can be expressed in terms of the Levi-Civita connection $\nabla^{M}$ through the following Gauss-type formula

$$
\left\{\begin{array}{l}
\nabla_{\xi}^{M} Z=\nabla_{\xi} Z+h(Z)-g(Z, \kappa) \xi, \\
\nabla_{Z}^{M} W=\nabla_{Z} W-g(h(Z), W) \xi,
\end{array}\right.
$$

where $\kappa:=\nabla_{\xi}^{M} \xi$ is the mean curvature of the flow and $Z, W$ are sections in $\Gamma(Q)$.

Since $T M=\mathbb{R} \xi \oplus Q$ the normal bundle $Q \rightarrow M$ is spin (as a vector bundle) if and only if $T M$ is. Therefore, if we assume that $M$ carries a spin structure, then its pull-back via the double covering defines a spin structure on $Q[3,5]$. In that case $M$ carries its own spinor bundle $\Sigma M$ as well as the spinor bundle of $Q$, denoted by $\Sigma Q$. Actually there exists 
a unitary isomorphism of Hermitian vector bundles (that we denote by the identity map $\varphi \mapsto \varphi)$

$$
\Sigma M \rightarrow \begin{cases}\Sigma Q & \text { if } n \text { is even, } \\ \Sigma Q \oplus \Sigma Q & \text { if } n \text { is odd }\end{cases}
$$

satisfying, for every $Z \in \Gamma(Q)$ and $\varphi \in \Gamma(\Sigma M)$

- W.r.t. the Clifford multiplications "." in $\Sigma M$ and "." in $\Sigma Q$ respectively

$$
\xi \cdot Z \cdot \varphi= \begin{cases}Z \cdot \varphi & \text { if } n \text { is even } \\ \left(Z_{Q} \cdot \oplus-Z \cdot\right) \varphi & \text { if } n \text { is odd. }\end{cases}
$$

- The Clifford action of $i \xi$ is given by

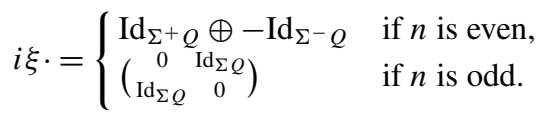

- W.r.t. the spinorial Levi-Civita connections $\nabla^{M}$ on $\Sigma M$ and $\nabla$ on $\Sigma Q$ one has [15, (2.4.7)]

$$
\left\{\begin{array}{l}
\nabla_{\xi}^{M} \varphi=\nabla_{\xi} \varphi+\frac{1}{2} \Omega \cdot \varphi+\frac{1}{2} \xi \cdot \kappa \cdot \varphi, \\
\nabla_{Z}^{M} \varphi=\nabla_{Z} \varphi+\frac{1}{2} \xi \cdot h(Z) \cdot \varphi .
\end{array}\right.
$$

In particular the covariant derivative $\nabla$ is metric on $\Sigma M$ : if $\langle\cdot, \cdot\rangle$ denotes a natural Hermitian inner product on $\Sigma M$, then $X\langle\varphi, \psi\rangle=\left\langle\nabla_{X} \varphi, \psi\right\rangle+\left\langle\varphi, \nabla_{X} \psi\right\rangle$ for all $X \in \Gamma(T M)$ and $\varphi, \psi \in$ $\Gamma(\Sigma M)$. It also follows from (2) that the Clifford action of $\xi$ is $\nabla$-parallel: $\nabla_{X}(\xi \cdot \varphi)=$ $\xi \cdot \nabla_{X} \varphi$ for every $X \in \Gamma(T M)$ and $\varphi \in \Gamma(\Sigma M)$. Therefore, if one defines $\Sigma_{+} M$ and $\Sigma_{-} M$ by

$$
\Sigma_{ \pm} M:=\operatorname{Ker}\left(i \xi \cdot \mp \operatorname{Id}_{\Sigma M}\right),
$$

then $\Sigma M$ splits into the orthogonal and $\nabla$-parallel direct sum $\Sigma M=\Sigma_{+} M \oplus \Sigma_{-} M$. Furthermore both $\Sigma_{+} M$ and $\Sigma_{-} M$ have the same rank since they are exchanged by the Clifford action of any non-zero section $Z \in \Gamma(Q)$. In the case where $n$ is even, one has $\Sigma_{ \pm} M=\Sigma^{ \pm} Q$, however in the case where $n$ is odd $\Sigma_{ \pm} M$ never coincides with one of the two copies of $\Sigma Q$ hence with one of the eigenspaces $\Sigma^{ \pm} M$ of the Clifford action of the complex volume form of $M$.

In the following almost all Riemannian flows under consideration will be minimal, i.e., $\kappa=0$. More precisely, we shall mainly deal with the following families of Riemannian flows, which of course are not disjoint from each other: the case where $\xi$ is the fundamental vector field of a free isometric $\mathbb{S}^{1}$-action with totally geodesic orbits, the case where $\xi$ is parallel, corresponding to local Riemannian products of a one-dimensional manifold with an $n$-dimensional one (this is also equivalent to $\kappa=0$ and $h=0$ ), and the case where $\xi$ is the Reeb vector field of a Sasakian manifold

Definition 2.1 A Riemannian manifold is called Sasakian if and only if it is a Riemannian flow $\left(M^{n+1}, g, \mathcal{F}\right)$ satisfying

(i) $\kappa=0$, i.e., the flow is minimal,

(ii) $h^{2}=-\operatorname{Id}_{Q}$, i.e., $h$ is an almost-Hermitian structure on $Q$,

(iii) $\nabla h=0$, i.e., $h$ is parallel on $Q$ (hence is a Kähler structure on $Q$ ). 
It can be easily checked that this definition is equivalent to the usual one, where one requires $\xi$ to be a unit Killing vector field satisfying

$$
\left\{\begin{array}{l}
\left(\nabla^{M} \xi\right)^{2}=-\mathrm{Id}_{T M}+\xi^{b} \otimes \xi, \\
\left(\nabla_{X}^{M} \nabla^{M} \xi\right)(Y)=g(\xi, Y) X-g(X, Y) \xi,
\end{array}\right.
$$

for all $X, Y \in \Gamma(T M)$. From Definition 2.1 the normal bundle $Q$ of any Sasakian manifold carries a canonical Kähler structure. In particular such a manifold is always odddimensional. We shall from now on denote $m:=\frac{n}{2}$. In the following we shall also omit to write $\mathcal{F}$ for the flow and consider a Sasakian manifold as a triple $\left(M^{2 m+1}, g, \xi\right)$.

If now a Sasakian manifold $M$ is spin, then the Clifford action of the 2-form $\Omega$ (which is then the Kähler form of $Q$ ) splits $\Sigma M$ into the following orthogonal and $\nabla$-parallel decomposition [17]:

$$
\Sigma M=\bigoplus_{r=0}^{m} \Sigma_{r} M
$$

where $\Sigma_{r} M$ is the eigenbundle associated with the eigenvalue $i(2 r-m)$ of $\Omega$ for every $r \in\{0, \ldots, m\}$. W.r.t. the Clifford action of $i \xi$ one has $\Sigma_{+} M=\bigoplus_{r \text { even }} \Sigma_{r} M$ and $\Sigma_{-} M=$ $\bigoplus_{r \text { odd }} \Sigma_{r} M$, that is $\left.i \xi\right|_{\Sigma_{r} M}=(-1)^{r} \operatorname{Id}_{\Sigma_{r} M}$ [13]. Moreover, the subspaces $\Sigma_{0} M$ and $\Sigma_{m} M$ can be characterized by the property that for all $Z$ orthogonal to $\xi$ we have

$$
h(Z) \cdot \psi_{0}=i Z \cdot \psi_{0} \text { and } h(Z) \cdot \psi_{m}=-i Z \cdot \psi_{m} .
$$

Replacing the metric by a positive scalar multiple of it obviously preserves the structure of Riemannian flow. There exists however a less trivial type of flow-preserving deformations of the metric that we will need in the next sections and which are called $\mathcal{D}$-homothetic:

Definition 2.2 (S. Tanno [23]) Let $\left(M^{n+1}, g, \mathcal{F}\right)$ be a Riemannian flow given by a unit vector field $\xi$. A $\mathcal{D}$-homothetic deformation of $g$ is a metric $\bar{g}_{t}$ on $M$ of the form

$$
\bar{g}_{t}:=t^{2} g_{\mid \mathbb{R} \xi}+t g_{\mid Q}
$$

for some real number $t>0$.

A $\mathcal{D}$-homothetic deformation of $g$ may be obtained as follows: first rescale $g$ by a factor $t$ in the direction of the flow, then multiply the obtained metric by $t$. It is first to be noticed that a $\mathcal{D}$-homothetic deformation of a Riemannian flow is again a Riemannian flow, more precisely:

Lemma 2.3 Let $\left(M^{n+1}, g, \mathcal{F}\right)$ be a Riemannian flow given by a unit vector field $\xi$ and $\bar{g}_{t}:=t^{2} g_{\left.\right|_{\mathbb{R} \xi}}+t g_{\left.\right|_{Q}}$ be a $\mathcal{D}$-homothetic deformation of $g$ with $t \in \mathbb{R}_{+}^{*}$. Then $\left(M^{n+1}, \bar{g}_{t}, \mathcal{F}\right)$ is a Riemannian flow described by the unit vector field $\bar{\xi}^{t}:=\frac{1}{t} \xi$. If furthermore $\bar{\xi}^{t}, \bar{\nabla}^{t}, \bar{h}^{t}, \bar{\kappa}^{t}$ denote the corresponding objects for $\bar{g}_{t}$, then the following holds:

(i) One has $\bar{\xi}^{t}=\frac{1}{t} \xi, \bar{h}^{t}=h$ and $\bar{\kappa}^{t}=\frac{1}{t} \kappa$.

(ii) On $Q$ one has $\bar{\nabla}^{t}=\nabla$.

(iii) If furthermore $M$ is spin, then there exists a unitary isomorphism

$$
\begin{gathered}
\Sigma_{g} M \longrightarrow \Sigma_{\bar{g}_{t}} M, \\
\varphi \longmapsto \bar{\varphi}^{t},
\end{gathered}
$$


s.t., if “-." denotes the Clifford multiplication on $\Sigma_{\bar{g}_{t}} M$,

- $\overline{\xi \cdot \varphi}=\bar{\xi}^{t} \cdot \bar{\varphi}^{t}$ and $\overline{Z \cdot \varphi}=\frac{1}{\sqrt{t}} Z \cdot \bar{\varphi}^{t}$ for every $Z \in \Gamma(Q)$.

- $\bar{\nabla}_{X}^{t} \bar{\varphi}^{t}={\overline{\nabla_{X} \varphi}}^{t}$ for every $X \in \Gamma(T M)$.

(iv) If $\left(M^{n+1}, g, \xi\right)$ is Sasakian, then so is $\left(M^{n+1}, \bar{g}_{t}, \bar{\xi}_{t}\right)$.

The proof of Lemma 2.3 consists of elementary computations and identifications that we leave to the reader.

\section{Transversal Killing spinors}

\subsection{Definition}

We generalize in some sense the Killing spinor equation (see e.g. [7, 16] for references on that topic) to the set up of Riemannian flows.

Definition 3.1 Let $\left(M^{n+1}, g, \mathcal{F}\right)$ be a spin Riemannian flow given by a unit vector field $\xi$. Let $\alpha, \beta \in \mathbb{C}$. An $(\alpha, \beta)$-transversal Killing spinor on $M$ is a smooth section $\psi$ of $\Sigma M$ satisfying, for all $Z \in \Gamma(Q)$,

$$
\left\{\begin{array}{l}
\nabla_{\xi} \psi=\alpha \xi \cdot \psi, \\
\nabla_{Z} \psi=\beta \xi \cdot Z \cdot \psi
\end{array}\right.
$$

If $\alpha=0$, then $\psi$ is called a basic $\beta$-Killing spinor (see also [15]), and if $\alpha=\beta=0$ it is called basic parallel or transversally parallel spinor.

First note that an $(\alpha, \beta)$-transversal Killing spinor is a parallel section of $\Sigma M$ w.r.t. the covariant derivative $\widetilde{\nabla}$ defined by

$$
\widetilde{\nabla}_{X} \varphi:=\nabla_{X} \varphi-\alpha g(X, \xi) \xi \cdot \varphi-\beta \xi \cdot X \cdot \varphi-\beta g(X, \xi) \varphi
$$

for all $X \in \Gamma(T M)$ and $\varphi \in \Gamma(\Sigma M)$. Hence if an $(\alpha, \beta)$-transversal Killing spinor vanishes at one point it vanishes everywhere on $M$.

\section{Notes 3.2}

1. If $\psi$ is an $(\alpha, \beta)$-transversal Killing spinor on $\left(M^{n+1}, g, \mathcal{F}\right)$, then $\xi \cdot \psi$ is an $(\alpha,-\beta)$ transversal Killing spinor. Therefore $\beta$ can always be changed into $-\beta$, independently of the dimension or the orientation of the manifold. This is in general not possible for $\alpha$, see e.g. Notes 4.9 .

2. Let $\psi$ be an $(\alpha, \beta)$-transversal Killing spinor and $\psi=\psi_{+}+\psi_{-}$its decomposition w.r.t. the Clifford action of $i \xi$ (i.e., $i \xi \cdot \psi_{ \pm}= \pm \psi_{ \pm}$). Then $\psi_{ \pm}$satisfies $\nabla_{\xi} \psi_{ \pm}=\alpha \xi \cdot \psi_{ \pm}$and $\nabla_{Z} \psi_{ \pm}=\beta \xi \cdot Z \cdot \psi_{\mp}$ for all $Z \in \Gamma(Q)$ (this follows from the action of $\xi$ being $\nabla$-parallel and from $\xi \cdot Z=-Z \cdot \xi$ for all $Z \in \Gamma(Q))$. Therefore $\psi_{ \pm}$is again a transversal Killing spinor only if $\beta=0$; if $\beta \neq 0$, then $\psi$ vanishes as soon as $\psi_{+}$or $\psi_{-}$vanishes on a non-empty open subset of $M$.

3. If $\alpha$ and $\beta$ are real, then any $(\alpha, \beta)$-transversal Killing spinor has constant length on $M$, since in that case it can be easily checked that the covariant derivative $\widetilde{\nabla}$ above is metric. 
4. In the particular case where $h=0$ and $\kappa=0$, i.e., $M$ carries a parallel unit vector field, then $(0, \beta)$-transversal Killing spinors for some $\beta \in \mathbb{R}$ are exactly the spinor fields defined by B. Alexandrov, G. Grantcharov and S. Ivanov in [1, (8)] and studied in [1, Theorem 3.1].

5. We notice that Th. Friedrich and E.C. Kim defined on a Sasakian manifold $M$ the notion of quasi-Killing spinor of type $(a, b)[13, \mathrm{p} .23]$ which are $a$-Killing (for the Levi-Civita connection on $M)$ in the direction of the normal bundle $Q$ and $(a+b)$-Killing in the direction of $\xi$. They show that the condition of the flow being $\eta$-Einstein is sufficient and necessary for the existence of such spinors. In that case and for a suitable choice of $a$ and $b$ (the product $a(a+b)$ could be not zero) they are solutions of the so-called EinsteinDirac equations. Here we point out that the notion of transversal Killing spinors is in general different from the quasi-Killing spinors since in our consideration it is Killing for the transversal connection.

6. A similar equation appears in [18, p. 137 (8.3)], where however the connection $\nabla$ denotes the Levi-Civita connection of $\Sigma M$ (in our notation it corresponds to $\nabla^{M}$ ).

In the following subsections we want to characterize those Riemannian flows that admit non-trivial $(\alpha, \beta)$-transversal Killing spinors. The following lemma follows straightforward from Lemma 2.3 .

Lemma 3.3 Let $\left(M^{n+1}, g, \mathcal{F}\right)$ be a spin Riemannian flow. For a fixed $t \in \mathbb{R}_{+}^{*}$ let $\bar{g}_{t}:=t^{2} g_{\xi}+$ $\operatorname{tg}_{\left.\right|_{Q}}$ be a $\mathcal{D}$-homothetic deformation of $g$. If $\psi$ is an $(\alpha, \beta)$-transversal Killing spinor on $\left(M^{n+1}, g, \mathcal{F}\right)$, then $\bar{\psi}^{t}$ is an $\left(\bar{\alpha}^{t}, \bar{\beta}^{t}\right)$-transversal Killing spinor on $\left(M^{n+1}, \bar{g}_{t}, \bar{\xi}^{t}:=\frac{1}{t} \xi\right)$ with $\bar{\alpha}^{t}:=\frac{\alpha}{t}$ and $\bar{\beta}^{t}:=\frac{\beta}{\sqrt{t}}$.

3.2 General integrability conditions for transversal Killing spinors

Theorem 3.4 Let $\left(M^{n+1}, g, \mathcal{F}\right)$ be a spin Riemannian flow given by a unit vector field $\xi$ carrying an $(\alpha, \beta)$-transversal Killing spinor $\psi$. Let $\mathrm{Ric}_{M}$ and $\mathrm{Scal}_{M}$ denote the Ricci tensor and the scalar curvature of $(M, g)$ respectively. Then for any local orthonormal basis $\left\{e_{j}\right\}_{1 \leq j \leq n}$ of $Q$ one has

$$
\begin{aligned}
\operatorname{Ric}_{M}(\xi) \cdot \psi= & \left(|h|^{2}-|\kappa|^{2}\right) \xi \cdot \psi+4 n \alpha \beta \psi+2 \alpha \xi \cdot \kappa \cdot \psi+\kappa \cdot \Omega \cdot \psi \\
& +4 h(\kappa) \cdot \psi-\sum_{j=1}^{n} \xi \cdot e_{j} \cdot \nabla_{e_{j}}^{M} \kappa \cdot \psi \\
& +\frac{1}{2} \sum_{j, k=1}^{n} e_{j} \cdot e_{k} \cdot \nabla_{e_{j}} h\left(e_{k}\right) \cdot \psi+\sum_{j=1}^{n} \xi \cdot e_{j} \cdot \nabla_{\xi} h\left(e_{j}\right) \cdot \psi
\end{aligned}
$$

and for every $Z \in \Gamma(Q)$,

$$
\begin{aligned}
\operatorname{Ric}_{M}(Z) \cdot \psi= & -4 \alpha(h(Z)+\beta Z) \cdot \xi \cdot \psi+2 h^{2}(Z) \cdot \psi+4(n-1) \beta^{2} Z \cdot \psi \\
& +\frac{1}{2} \sum_{j=1}^{n} \xi \cdot e_{j} \cdot \nabla_{Z} h\left(e_{j}\right) \cdot \psi-\sum_{j=1}^{n} \xi \cdot e_{j} \cdot \nabla_{e_{j}} h(Z) \cdot \psi \\
& -\nabla_{\xi} h(Z) \cdot \psi+g(Z, \kappa)(-2 \alpha \psi+\xi \cdot \Omega \cdot \psi-\kappa \cdot \psi) \\
& +\nabla_{Z}^{M} \kappa \cdot \psi-h(Z) \cdot \xi \cdot \kappa \cdot \psi .
\end{aligned}
$$


Furthermore, one has

$$
\begin{aligned}
\operatorname{Scal}_{M} \psi= & \left(4 n(n-1) \beta^{2}-|h|^{2}-2|\kappa|^{2}\right) \psi-8 n \alpha \beta \xi \cdot \psi+8 \alpha \xi \cdot \Omega \cdot \psi \\
& -\sum_{j, k=1}^{n} \xi \cdot e_{j} \cdot e_{k} \cdot \nabla_{e_{k}} h\left(e_{j}\right) \cdot \psi+2 \sum_{j=1}^{n} e_{j} \cdot \nabla_{\xi} h\left(e_{j}\right) \cdot \psi \\
& +4 \alpha \kappa \cdot \psi+2 \xi \cdot \kappa \cdot \Omega \cdot \psi-2 \sum_{j=1}^{n} e_{j} \cdot \nabla_{e_{j}}^{M} \kappa \cdot \psi
\end{aligned}
$$

Proof Plugging (5) in (2) gives with the use of (1) that for $Z, W \in \Gamma(Q)$

$$
\begin{aligned}
\nabla_{Z}^{M} \nabla_{W}^{M} \psi= & \nabla_{Z}^{M}\left(\beta \xi \cdot W \cdot \psi+\frac{1}{2} \xi \cdot h(W) \cdot \psi\right) \\
= & \beta \xi \cdot \nabla_{Z} W \cdot \psi+\frac{1}{2} \xi \cdot h\left(\nabla_{Z} W\right) \cdot \psi+\frac{1}{2} \xi \cdot \nabla_{Z} h(W) \cdot \psi \\
& +\frac{\beta}{2}(h(Z) \cdot W \cdot \psi+h(W) \cdot Z \cdot \psi) \\
& +\frac{1}{4} h(Z) \cdot h(W) \cdot \psi+\beta^{2} W \cdot Z \cdot \psi
\end{aligned}
$$

By the fact that the torsion of $\nabla^{M}$ is zero we get from (1) that $[Z, W]=\nabla_{Z} W-\nabla_{W} Z-$ $2 g(h(Z), W) \xi$ so that

$$
\begin{aligned}
\nabla_{[Z, W]}^{M} \psi= & \beta \xi \cdot\left(\nabla_{Z} W-\nabla_{W} Z\right) \cdot \psi+\frac{1}{2} \xi \cdot h\left(\nabla_{Z} W-\nabla_{W} Z\right) \cdot \psi \\
& -2 g(h(Z), W)\left(\alpha \xi \cdot \psi+\frac{1}{2} \Omega \cdot \psi+\frac{1}{2} \xi \cdot \kappa \cdot \psi\right) .
\end{aligned}
$$

We deduce that

$$
\begin{aligned}
R_{Z, W}^{M} \psi= & \nabla_{[Z, w]}^{M} \psi-\left[\nabla_{Z}^{M}, \nabla_{W}^{M}\right] \psi \\
= & -2 g(h(Z), w)\left(\alpha \xi \cdot \psi+\frac{1}{2} \Omega \cdot \psi+\frac{1}{2} \xi \cdot \kappa \cdot \psi\right) \\
& -\frac{1}{2} \xi \cdot\left(\nabla_{Z} h(W)-\nabla_{W} h(Z)\right) \cdot \psi \\
& +\frac{1}{4}(h(W) \cdot h(Z) \cdot \psi-h(Z) \cdot h(W) \cdot \psi)+\beta^{2}(Z \cdot W \cdot \psi-W \cdot Z \cdot \psi) .
\end{aligned}
$$

On the other hand since $[\Omega, Z]=2 h(Z)$, one has

$$
\begin{aligned}
\nabla_{Z}^{M} \nabla_{\xi}^{M} \psi= & \nabla_{Z}^{M}\left(\alpha \xi \cdot \psi+\frac{1}{2} \Omega \cdot \psi+\frac{1}{2} \xi \cdot \kappa \cdot \psi\right) \\
= & \frac{\alpha}{2} h(Z) \cdot \psi-\alpha \beta Z \cdot \psi+\frac{1}{2} \nabla_{Z}^{M} \Omega \cdot \psi+\frac{\beta}{2} \xi \cdot Z \cdot \Omega \cdot \psi \\
& +\beta \xi \cdot h(Z) \cdot \psi+\frac{1}{4} \xi \cdot h(Z) \cdot \Omega \cdot \psi+\frac{1}{2} \xi \cdot h^{2}(Z) \cdot \psi \\
& +\frac{1}{4} h(Z) \cdot \kappa \cdot \psi-\frac{1}{2} g(h(Z), \kappa) \psi+\frac{\beta}{2} \kappa \cdot Z \cdot \psi+\frac{1}{2} \xi \cdot \nabla_{Z}^{M} \kappa \cdot \psi
\end{aligned}
$$


and, still using (1),

$$
\begin{aligned}
\nabla_{\xi}^{M} \nabla_{Z}^{M} \psi= & \nabla_{\xi}^{M}\left(\beta \xi \cdot Z \cdot \psi+\frac{1}{2} \xi \cdot h(Z) \cdot \psi\right) \\
= & \beta \xi \cdot \nabla_{\xi} Z \cdot \psi+\frac{1}{2} \xi \cdot h\left(\nabla_{\xi} Z\right) \cdot \psi+\frac{\beta}{2} \kappa \cdot Z \cdot \psi \\
& +\beta \xi \cdot h(Z) \cdot \psi+\alpha \beta Z \cdot \psi+\frac{\beta}{2} \xi \cdot Z \cdot \Omega \cdot \psi \\
& +\frac{1}{4} \kappa \cdot h(Z) \cdot \psi+\frac{1}{2} \xi \cdot \nabla_{\xi} h(Z) \cdot \psi+\frac{1}{2} \xi \cdot h^{2}(Z) \cdot \psi \\
& +\frac{\alpha}{2} h(Z) \cdot \psi+\frac{1}{4} \xi \cdot h(Z) \cdot \Omega \cdot \psi .
\end{aligned}
$$

Moreover by the vanishing of the torsion on $M$ we get $[Z, \xi]=-\nabla_{\xi} Z+g(Z, \kappa) \xi$. We deduce that

$$
\begin{aligned}
R_{Z, \xi}^{M} \psi= & -\nabla_{\nabla_{\xi} Z}^{M} \psi+g(Z, \kappa) \nabla_{\xi}^{M} \psi-\left[\nabla_{Z}^{M}, \nabla_{\xi}^{M}\right] \psi \\
= & g(Z, \kappa)\left(\alpha \xi \cdot \psi+\frac{1}{2} \Omega \cdot \psi+\frac{1}{2} \xi \cdot \kappa \cdot \psi\right)+2 \alpha \beta Z \cdot \psi-\frac{1}{2} \nabla_{Z}^{M} \Omega \cdot \psi \\
& -\frac{1}{2} h(Z) \cdot \kappa \cdot \psi-\frac{1}{2} \xi \cdot \nabla_{Z}^{M} \kappa \cdot \psi+\frac{1}{2} \xi \cdot \nabla_{\xi} h(Z) \cdot \psi
\end{aligned}
$$

Recalling that from its definition $\Omega$ satisfies $\Omega(\xi, \cdot)=0$ one can compute the Clifford action of $\nabla^{M} \Omega$ and find that for every $Z \in \Gamma(Q)$,

$$
\nabla_{Z}^{M} \Omega \cdot \psi=h^{2}(Z) \cdot \xi \cdot \psi+\frac{1}{2} \sum_{j=1}^{n} e_{j} \cdot \nabla_{Z} h\left(e_{j}\right) \cdot \psi .
$$

We can hence rewrite

$$
\begin{aligned}
R_{Z, \xi}^{M} \psi= & g(Z, \kappa)\left(\alpha \xi \cdot \psi+\frac{1}{2} \Omega \cdot \psi+\frac{1}{2} \xi \cdot \kappa \cdot \psi\right)+2 \alpha \beta Z \cdot \psi-\frac{1}{2} h^{2}(Z) \cdot \xi \cdot \psi \\
& -\frac{1}{2} h(Z) \cdot \kappa \cdot \psi-\frac{1}{2} \xi \cdot \nabla_{Z}^{M} \kappa \cdot \psi+\frac{1}{2} \xi \cdot \nabla_{\xi} h(Z) \cdot \psi \\
& -\frac{1}{4} \sum_{j=1}^{n} e_{j} \cdot \nabla_{Z} h\left(e_{j}\right) \cdot \psi
\end{aligned}
$$

Applying [16, p. 156] for the local orthonormal frame $\left\{e_{j}\right\}_{1 \leq j \leq n+1}$ of $T M$ with $e_{n+1}=\xi$ we obtain

$$
\begin{aligned}
\operatorname{Ric}_{M}(Z) \cdot \psi= & 2 \sum_{j=1}^{n+1} e_{j} \cdot R_{Z, e_{j}}^{M} \psi \\
= & -4 \alpha(h(Z)+\beta Z) \cdot \xi \cdot \psi+2 h^{2}(Z) \cdot \psi-h(Z) \cdot \xi \cdot \kappa \cdot \psi \\
& +\frac{1}{2} \sum_{k=1}^{n} \xi \cdot e_{k} \cdot \nabla_{Z} h\left(e_{k}\right) \cdot \psi-\sum_{k=1}^{n} \xi \cdot e_{k} \cdot \nabla_{e_{k}} h(Z) \cdot \psi-\nabla_{\xi} h(Z) \cdot \psi \\
& +4(n-1) \beta^{2} Z \cdot \psi+g(Z, \kappa)(-2 \alpha \psi+\xi \cdot \Omega \cdot \psi-\kappa \cdot \psi)+\nabla_{Z}^{M} \kappa \cdot \psi
\end{aligned}
$$


This shows the second identity of Theorem 3.4. On the other hand

$$
\begin{aligned}
\operatorname{Ric}_{M}(\xi) \cdot \psi= & 2 \alpha \xi \cdot \kappa \cdot \psi+\kappa \cdot \Omega \cdot \psi+4 h(\kappa) \cdot \psi+\left(|h|^{2}-|\kappa|^{2}\right) \xi \cdot \psi+4 n \alpha \beta \psi \\
& +\frac{1}{2} \sum_{j, k=1}^{n} e_{j} \cdot e_{k} \cdot \nabla_{e_{j}} h\left(e_{k}\right) \cdot \psi-\sum_{j=1}^{n} \xi \cdot e_{j} \cdot \nabla_{e_{j}}^{M} \kappa \cdot \psi \\
& +\sum_{j=1}^{n} \xi \cdot e_{j} \cdot \nabla_{\xi} h\left(e_{j}\right) \cdot \psi .
\end{aligned}
$$

This shows the first identity of Theorem 3.4. We compute now the action of the scalar curvature of $M$ :

$$
\begin{aligned}
\operatorname{Scal}_{M} \psi= & -\sum_{j=1}^{n+1} e_{j} \cdot \operatorname{Ric}_{M}\left(e_{j}\right) \cdot \psi \\
= & \left(4 n(n-1) \beta^{2}-|h|^{2}-2|\kappa|^{2}\right) \psi-8 n \alpha \beta \xi \cdot \psi+8 \alpha \xi \cdot \Omega \cdot \psi \\
& -\sum_{j, k=1}^{n} \xi \cdot e_{j} \cdot e_{k} \cdot \nabla_{e_{k}} h\left(e_{j}\right) \cdot \psi+2 \sum_{j=1}^{n} e_{j} \cdot \nabla_{\xi} h\left(e_{j}\right) \cdot \psi \\
& +4 \alpha \kappa \cdot \psi+2 \xi \cdot \kappa \cdot \Omega \cdot \psi-2 \sum_{j=1}^{n} e_{j} \cdot \nabla_{e_{j}}^{M} \kappa \cdot \psi .
\end{aligned}
$$

This shows the third identity and achieves the proof of Theorem 3.4.

Note 3.5 Under the hypotheses of Theorem 3.4, if one furthermore assumes that $\psi$ is a nonzero $(\alpha, \beta)$-transversal Killing spinor with real $\alpha$ and $\beta$, that $\kappa=0$ and $\nabla h=0$ (e.g. if $M$ is a local Riemannian product or if $M$ is Sasakian) then necessarily $\alpha \beta=0$. From Theorem 3.4 the scalar curvature of $M$ must indeed satisfy the condition

$$
\operatorname{Scal}_{M} \psi=\left(4 n(n-1) \beta^{2}-|h|^{2}\right) \psi-8 n \alpha \beta \xi \cdot \psi+8 \alpha \xi \cdot \Omega \cdot \psi,
$$

where $\psi$ is the $(\alpha, \beta)$-transversal Killing spinor on $M$. Taking the Hermitian product with $\xi \cdot \psi$ and identifying the real parts one obtains $0=-8 n \alpha \beta|\psi|^{2}$. Since $\psi$ does not vanish identically we deduce that $\alpha \beta=0$.

\subsection{Examples of transversal Killing spinors}

We construct a first important family of examples of Riemannian flows with transversal Killing spinors. Recall for the next proposition that the unit circle $\mathbb{S}^{1}$ carries two different spin structures, the trivial one that we call $(\delta=0)$-spin structure and the non-trivial one that we call $(\delta=1)$-spin structure. We also recall that a $\beta$-Killing spinor on a Riemannian spin manifold $N^{n}$ is a section $\psi$ of $\Sigma N$ satisfying

$$
\nabla_{X}^{N} \psi=\beta X_{N} \psi
$$

for every $X \in \Gamma(T N)$. If a non-zero such spinor field exists, then $N^{n}$ is Einstein with scalar curvature $4 n(n-1) \beta^{2}$ (see e.g. [16, Proposition 5.12] or [7]), hence $\beta$ must be either real or purely imaginary. The classification of the Riemannian spin manifolds with non-trivial $\beta$-Killing spinors was achieved in $[4,6,25]$. 
Proposition 3.6 Let $N$ be an $n$-dimensional Riemannian spin manifold carrying a $\beta$-Killing spinor $\psi$ for some $\beta \in \mathbb{C}$ and $M \stackrel{\pi}{\longrightarrow} N$ be a Riemannian submersion which is either an $\mathbb{S}^{1}$-bundle with totally geodesic fibres over $N$ or the second projection of the Riemannian product $M:=\mathbb{R} \times N$ onto $N$. Let $M$ be endowed with the spin structure induced by that of $N$ and the trivial spin structure on $\mathbb{S}^{1}$ or $\mathbb{R}$ respectively. Then the following holds:

(i) The spinor $\psi$ on $N$ induces a $(0, \beta)$-transversal Killing spinor on $M$.

(ii) In the second case $(M=\mathbb{R} \times N)$ if moreover $\beta=0$ then $\psi$ induces an $(\alpha, 0)$-transversal Killing spinor on $M$ for any $\alpha \in \mathbb{C}$. Furthermore for $\alpha \in \mathbb{R}$ the spinor field $\psi$ descends to the Riemannian product $\mathbb{S}^{1} \times N$ if and only if $\alpha \in \frac{\pi \delta}{L}+\frac{2 \pi \mathbb{Z}}{L}$, where $L$ is the length of the unit circle.

Proof We recall the following lemma about spinors on submersions and $\mathbb{S}^{1}$-bundles, see [18, Chap. 1] or [3]:

Lemma 3.7 Let $M \stackrel{\pi}{\longrightarrow} N$ be as in Proposition 3.6. Then the following statements hold:

1. The manifold $M$ defines a minimal spin Riemannian flow w.r.t. the unit fundamental vector field $\xi$ given by the $\mathbb{S}^{1}$-action or $\frac{\partial}{\partial t}$ respectively and carries a spin structure which is induced by those of $N$ and the trivial one on $\mathbb{S}^{1}$ or $\mathbb{R}$ respectively.

2. The spinor bundle of $Q$ can be identified with $\pi^{*} \Sigma N$, in particular $\xi \cdot X^{*} \cdot \varphi=X_{N} \varphi$ for every $X \in \Gamma(T N)$, where $X^{*} \in \Gamma(Q)$ denotes the horizontal lift of $X$ to $M$.

3. For every $\varphi \in \Gamma(\Sigma N)$ (which is identified to $\varphi \circ \pi \in \Gamma\left(\pi^{*} \Sigma N\right)$ ) one has

$$
\left\{\begin{array}{l}
\nabla_{X^{*}} \varphi=\nabla_{X}^{N} \varphi \\
\nabla_{\xi} \varphi=0
\end{array}\right.
$$

for every $X \in \Gamma(T N)$. Besides a spinor $\phi$ on $M$ is projectable on $N$ if and only if $\nabla_{\xi} \phi=0$.

Since $\psi$ is a $\beta$-Killing spinor on the base manifold $N$, then we deduce from Lemma 3.7 that it satisfies

$$
\left\{\begin{array}{l}
\nabla_{Z} \psi=\nabla_{Z}^{N} \psi=\beta Z_{N} \psi=\beta \xi \cdot Z \cdot \psi, \\
\nabla_{\xi} \psi=0,
\end{array}\right.
$$

for every $Z \in \Gamma\left(\pi^{*}(T N)\right) \cong Q$, hence $\psi$ is a $(0, \beta)$-transversal Killing spinor on $M$. Note that in the case $n$ odd we identify $\psi$ as a section of the first component $\Sigma Q$ of $\Sigma M$. This shows $i$. Assume now that $M:=\mathbb{R} \times N$ and $\beta=0$. For any $\alpha \in \mathbb{C}$ we set

$$
\phi:= \begin{cases}e^{-i \alpha t} \psi_{+}+e^{i \alpha t} \psi_{-} & \text {if } n \text { is even, } \\ e^{-i \alpha t}(\psi \oplus \psi) & \text { if } n \text { is odd, }\end{cases}
$$

where, if $n$ is even, $\psi=\psi_{+}+\psi_{-}$is the decomposition of $\psi$ w.r.t. the Clifford action of $i \xi$, see above. We check that, under the hypotheses of Proposition 3.6, the spinor $\phi$ is an $(\alpha, 0)$-transversal Killing spinor on $M$. We just describe the case $n$ even, the case $n$ odd being completely analogous. Since $\psi_{+}$and $\psi_{-}$are parallel, as a consequence of $\psi$ being parallel, then for all $Z \in \Gamma(Q)$ we deduce $\nabla_{Z} \phi=0$ which is $\beta \xi \cdot Z \cdot \phi$. Moreover

$$
\begin{aligned}
\nabla_{\xi} \phi & =i \alpha\left(-e^{-i \alpha t} \psi_{+}+e^{i \alpha t} \psi_{-}\right) \\
& =i \alpha(-i \xi \cdot)\left(e^{-i \alpha t} \psi_{+}+e^{i \alpha t} \psi_{-}\right) \\
& =\alpha \xi \cdot \phi
\end{aligned}
$$


hence $\phi$ is an $(\alpha, 0)$-transversal Killing spinor. Finally, let $M:=\mathbb{S}^{1} \times N$, where $\mathbb{S}^{1}$ carries the (left-, right- or bi-)invariant metric for which Length $\left(\mathbb{S}^{1}\right)=L>0$ and the $\delta$-spin structure (where $\delta \in\{0,1\}$ ). If $\alpha$ is real, then the spinor $\phi$ constructed above on $\mathbb{R} \times N$ satisfies the equivariance condition $\phi_{(t, x)}=e^{i \pi \delta} \phi_{(t-L, x)}$ for every $(t, x) \in \mathbb{R} \times N$ if and only if $e^{i \pi \delta} e^{-i \alpha L}=1$. In other words, $\phi$ descends to an $(\alpha, 0)$-transversal Killing spinor on $\mathbb{S}^{1} \times N$ for the metric and spin structure above if and only if $\alpha \in \frac{\pi \delta}{L}+\frac{2 \pi \mathbb{Z}}{L}$. This shows (ii) and achieves the proof of Proposition 3.6.

\section{Examples 3.8}

1. The most simple examples that come in mind as application of Proposition 3.6 are the Euclidean space and any flat torus. Since they admit w.r.t. their trivial spin structure parallel spinors [12], applying Proposition 3.6 one deduces that they carry an $(\alpha, 0)$ transversal Killing spinor for a suitable choice of $\alpha$.

2. In the same way any Riemannian product $\mathbb{R} \times \mathbb{S}^{n}$ or $\mathbb{S}^{1} \times \mathbb{S}^{n}$ (for $n>1$ ), where $\mathbb{S}^{n}$ carries its canonical metric and $\mathbb{S}^{1}$ with its trivial spin structure, admits $\left(0, \pm \frac{1}{2}\right)$-transversal Killing spinors.

3. Any $\mathbb{S}^{1}$-bundle which is also a Riemannian submersion with totally geodesic fibres over $\mathbb{S}^{n}$ carrying its canonical metric of sectional curvature 1 admits $\left(0, \pm \frac{1}{2}\right)$-transversal Killing spinors.

4. As a particular case of the preceding example consider the Hopf-fibration $\mathbb{S}^{3} \stackrel{\pi}{\longrightarrow} \mathbb{C P}^{1}$, where $\mathbb{S}^{3}$ is the 3-dimensional Euclidean sphere and $\mathbb{C P}^{1}$ is the complex projective space with its Fubini-Study metric of constant holomorphic sectional curvature 4. It is wellknown that $\pi$ is a $\mathbb{S}^{1}$-bundle and a Riemannian submersion with totally geodesic fibres. Furthermore the spin structure on $\mathbb{S}^{3}$ induced by those of $\mathbb{C P}$ and the trivial spin structure on $\mathbb{S}^{1}$ is its standard spin structure since there is only one spin structure on $\mathbb{S}^{3}$. Identifying $\mathbb{C P}^{1}$ with the 2-dimensional sphere $\mathbb{S}^{2}$ together with $\frac{1}{4}$ can we have a 2-dimensional space of \pm 1 -Killing spinors on $\mathbb{C P}^{1}$. We deduce from Proposition 3.6 that $\mathbb{S}^{3}$ carries a 2-dimensional space of $(0, \pm 1)$-transversal Killing spinors.

More generally, every lens space $\mathbb{Z}_{k} \backslash \mathbb{S}^{3}$ with its canonical metric and its trivial spin structure is also a $\mathbb{S}^{1}$-bundle with totally geodesic fibres over $\mathbb{C P}^{1}$, therefore it admits a non-zero $(0, \pm 1)$-transversal Killing spinor.

5. Let

$$
G:=\left\{\left(\begin{array}{lll}
1 & x & z \\
0 & 1 & y \\
0 & 0 & 1
\end{array}\right), x, y, z \in \mathbb{R}\right\}
$$

be the Heisenberg group, which is a 3-dimensional non-compact connected non-Abelian Lie group. For a fixed $r \in(\mathbb{Z} \backslash\{0\})$ consider the discrete subgroup $\Gamma_{r}$ of $G$ defined by

$$
\Gamma_{r}:=\left\{\left(\begin{array}{ccc}
1 & r x & z \\
0 & 1 & y \\
0 & 0 & 1
\end{array}\right), x, y, z \in \mathbb{Z}\right\} .
$$

The (homogeneous) quotient $M_{r}:=\Gamma_{r} \backslash G$ is a compact 3-dimensional manifold called a Heisenberg manifold. It carries a two-parameter family of left-invariant Riemannian metrics which make it into a Riemannian $\mathbb{S}^{1}$-principal bundle with totally geodesic fibres over a flat two-dimensional torus $\mathbb{T}^{2}:=r \mathbb{Z} \oplus \mathbb{Z} \backslash \mathbb{R}^{2}$ [3]. Fixing a flat metric and the trivial spin structure on $\mathbb{T}^{2}$ we have a 2-dimensional space of parallel spinors on $\mathbb{T}^{2}$. Hence it follows from Proposition 3.6 that, for the induced metric $g$ and the induced spin 
structure on $M_{r}$, there exists a 2-dimensional space of $(0,0)$-transversal Killing spinors on $\left(M_{r}, g\right)$. This has been already proved by G. Habib in [15] where the author performs a direct computation.

6. Let $M:=\widetilde{\mathrm{PSL}_{2}(\mathbb{R})}$ be the universal covering of the projective special linear group of $\mathbb{R}^{2}$. It can be identified with the unitary tangent bundle (or, equivalently, the bundle of positively-oriented orthonormal frames) $\mathbb{U} H^{2}$ of the hyperbolic plane $H^{2}$. Fixing the canonical metric and spin structure on $H^{2}$ we have a 2-dimensional space of $\pm i$-Killing spinors on $H^{2}$. From Proposition 3.6 we deduce that, for the induced metric and spin structure on $M$ we have a 2-dimensional space of $(0, \pm i)$-transversal Killing spinors on $M$.

\section{Transversal Killing spinors on Sasakian manifolds}

4.1 Integrability conditions for transversal Killing spinors on Sasakian manifolds

Let $\left(M^{2 m+1}, g, \xi\right)$ be a Sasakian manifold, see Definition 2.1. First note that, if $\psi$ is an $(\alpha, 0)$-transversal Killing spinor on a spin Sasakian manifold $\left(M^{2 m+1}, g\right)$, then so is every component $\psi_{r}$ of $\psi$ under the Clifford action of $\Omega$ (indeed the Clifford action of $\xi$ preserves $\psi_{r}$ and $\nabla \Omega=0$ ), compare with Notes 3.2.2.

Recall for the following corollary that a Riemannian flow is called $\eta$-Einstein [19] if and only if there exist real constants $\lambda, \mu$ on $M$ such that $\operatorname{Ric}_{M}=\lambda \operatorname{Id}_{T M}+\mu \xi^{b} \otimes \xi$.

Proposition 4.1 Under the hypotheses of Theorem 3.4, assume furthermore that $\left(M^{2 m+1}\right.$, $g, \xi)$ is Sasakian, that $\psi \neq 0$ and that $\alpha$ and $\beta$ are real. Then the following holds:

(i) Either $\alpha=0$ or $\beta=0$. If $\alpha \neq 0$ then either $\psi$ is an eigenspinor for the Clifford action by $\Omega$ or $m$ is odd and $\psi=\psi_{r}+\psi_{m-r}$ for some $r \in\{0, \ldots, m\}$.

(ii) If $\alpha=0$ then $\left(M^{2 m+1}, g\right.$, $\left.\xi\right)$ is $\eta$-Einstein.

(iii) If $\alpha \neq 0$ then $g\left(\operatorname{Ric}_{M}(Z), h(Z)\right)=0$ for every $Z \in \Gamma(Q)$. If furthermore $\psi_{0} \neq 0$ or $\psi_{m} \neq 0$ then $\left(M^{2 m+1}, g, \xi\right)$ is $\eta$-Einstein. This happens in particular if $\operatorname{dim}(M)=3$.

Proof Since on a Sasakian manifold $\kappa=0$ and $\nabla h=0$, we first deduce from Note 3.5 that $\alpha \beta=0$. The identities proved in Theorem 3.4 then simplify to

$$
\left\{\begin{array}{l}
\operatorname{Ric}_{M}(Z) \cdot \psi=-4 \alpha(h(Z)+\beta Z) \cdot \xi \cdot \psi+\left(4(2 m-1) \beta^{2}-2\right) Z \cdot \psi, \\
\operatorname{Ric}_{M}(\xi) \cdot \psi=2 m \xi \cdot \psi \\
\operatorname{Scal}_{M} \psi=2 m\left(4(2 m-1) \beta^{2}-1\right) \psi+8 \alpha \xi \cdot \Omega \cdot \psi
\end{array}\right.
$$

for every $Z \in \Gamma(Q)$. On every Sasakian manifold one has $\operatorname{Ric}_{M}(\xi)=2 m \xi$ [10] hence the second equation above is trivial. Consider now the last equation involving the scalar curvature of $M$. Decompose $\psi=\sum_{r=0}^{m} \psi_{r}$ according to (3) one obtains with the use of $\xi \cdot \psi_{r}=(-1)^{r+1} i \psi_{r}$ for every $r \in\{0, \ldots, m\}$ that

$$
\mathrm{Scal}_{M} \psi_{r}=2 m\left(4(2 m-1) \beta^{2}-1\right) \psi_{r}+(-1)^{r} 8 \alpha(2 r-m) \psi_{r} .
$$

We consider two cases:

- If $\alpha=0$ then coming back to the first equation in (6) we obtain

$$
\operatorname{Ric}_{M}(Z)=\left(4(2 m-1) \beta^{2}-2\right) Z,
$$

for every $Z \in \Gamma(Q)$, hence $\left(M^{2 m+1}, g, \xi\right)$ is $\eta$-Einstein and (ii) is proved. 
- If $\alpha \neq 0$ then $\beta=0$ and one obtains from (7)

$$
\mathrm{Scal}_{M}=-2 m+(-1)^{r} 8 \alpha(2 r-m)
$$

for every $r \in\{0, \ldots, m\}$ for which $\psi_{r}$ does not vanish. If there is more than one such $r$, say $r^{\prime}$, then one has in particular $(-1)^{r}(2 r-m)=(-1)^{r^{\prime}}\left(2 r^{\prime}-m\right)$. If $r+r^{\prime} \equiv 0$ (2) then $r=r^{\prime}$, contradiction, hence $r+r^{\prime} \equiv 1$ (2), from which one deduces that $r+r^{\prime}=m$. Therefore such an $r^{\prime}$ must then be unique (equal to $m-r$ ) and $m$ should be odd. We have proved (i).

As for the Ricci tensor on $Q$ in that case, we have the equation

$$
\operatorname{Ric}_{M}(Z) \cdot \psi_{r}=4(-1)^{r} i \alpha h(Z) \cdot \psi_{r}-2 Z \cdot \psi_{r}
$$

for every $Z \in \Gamma(Q)$ and every $r \in\{0, \ldots, m\}$ for which $\psi_{r}$ does not vanish. Taking the Hermitian product of that equation with $h(Z) \cdot \psi_{r}$ and identifying the real parts one obtains $g\left(\operatorname{Ric}_{M}(Z), h(Z)\right)=0$, and this holds for every $Z \in \Gamma(Q)$. Now if one furthermore assumes that $r=0$ or $r=m$, then with the use of (4) one deduces from (8) that, in the case $\psi_{0} \neq 0$, that $\operatorname{Ric}_{M}(Z)=(-2-4 \alpha) Z$, and in the case $\psi_{m} \neq 0$, that $\operatorname{Ric}_{M}(Z)=\left(-2+4(-1)^{m} \alpha\right) Z$. Hence the flow is $\eta$-Einstein in that case as well. Note that, if $m$ is odd, then both last expressions of the Ricci tensor are the same, which one could expect since in that case both $\psi_{0}$ and $\psi_{m}$ could be non-vanishing sections.

For $m=1$ the only possible values of $r$ are 0 and 1, hence the flow must always be $\eta$-Einstein. This shows (iii) and achieves the proof.

Note 4.2 Consider a Heisenberg manifold $\left(M_{r}, g, \xi\right)$ with metric and spin structure as in Examples 3.8. It is a Sasakian manifold. We have proved in Examples 3.8.5 that $\left(M_{r}, g, \xi\right)$ admits transversally parallel spinors. Actually there is no (non-zero) $(\alpha, \beta)$-transversal Killing spinors on $\left(M_{r}, g, \xi\right)$ for real $(\alpha, \beta) \neq(0,0)$. Assume indeed that $\psi$ were such a spinor field. If $\beta \neq 0$ then $\alpha=0$ and from Lemma 3.7 the spinor field $\psi$ would descend to a $\beta$ Killing spinor on a flat two-torus with trivial spin structure, contradiction. If $\alpha \neq 0$ then using $\operatorname{Ric}_{M}=-2 \mathrm{Id}_{T M}+4 \xi^{b} \otimes \xi$ on $\left(M_{r}, g, \xi\right)$ one would straightforward deduce from (6) that $\alpha h(Z) \cdot \xi \cdot \psi=0$ for every $Z \in \Gamma(Q)$, contradiction. Therefore $\alpha$ and $\beta$ necessarily vanish.

\subsection{Killing vs. transversal Killing spinors}

We now establish a relation between transversal and "classical" Killing spinors on Sasakian manifolds. Recall that a $\mathcal{D}$-homothetic deformation of a given metric $g$ on a Riemannian flow $(M, g, \mathcal{F})$ is a metric of the form $\bar{g}_{t}:=t^{2} g_{\mathbb{R}_{\xi}}+t g_{\left.\right|_{Q}}$ for some real number $t>0$.

Proposition 4.3 Let $\left(M^{2 m+1}, g, \xi\right)$ be a spin Sasakian manifold.

(a) The space of $-\frac{1}{2}$-Killing spinors in $\Sigma_{0} M$ coincides with that of $\left(-\frac{m+1}{2}, 0\right)$-transversal Killing spinors in $\Sigma_{0} M$. In particular, a section $\psi_{0}$ of $\Sigma_{0} M$ is an $(\alpha, 0)$-transversal Killing spinor on $\left(M^{2 m+1}, g, \xi\right)$ for some $\alpha<0$ if and only if there exists a $\mathcal{D}$-homothetic deformation $\bar{g}_{t}$ of $g$ for which the corresponding spinor field ${\overline{\psi_{0}}}^{t}$ is a- $\frac{1}{2}$-Killing spinor.

(b) The space of $\frac{(-1)^{m}}{2}$-Killing spinors in $\Sigma_{m} M$ coincides with that of $\left((-1)^{m} \frac{m+1}{2}, 0\right)$ transversal Killing spinors in $\Sigma_{m} M$. In particular, a section $\psi_{m}$ of $\Sigma_{m} M$ is an $(\alpha, 0)$ transversal Killing spinor on $\left(M^{2 m+1}, g, \xi\right)$ for some $\alpha$ such that $(-1)^{m} \alpha>0$ if and only if there exists a $\mathcal{D}$-homothetic deformation $\bar{g}_{t}$ of $g$ for which the corresponding spinor field ${\overline{\psi_{m}}}^{t}$ is a $\frac{(-1)^{m}}{2}$-Killing spinor. 
Proof First remember that, if $\beta=0$, then every component $\psi_{r}$ of $\psi$ is again an $(\alpha, 0)$ transversal Killing spinor on $M$, therefore we may talk about transversal Killing spinors lying in one of the components $\Sigma_{r} M$ of $\Sigma M$. Using (2) we compare $\nabla^{M} \varphi_{r}$ with $\nabla \varphi_{r}$ for any section $\varphi_{r}$ of $\Sigma_{r} M$ : on the one hand

$$
\begin{aligned}
\nabla_{\xi}^{M} \varphi_{r} & =\nabla_{\xi} \varphi_{r}+\frac{1}{2} \Omega \cdot \varphi_{r}+\frac{1}{2} \xi \cdot \kappa \cdot \varphi_{r} \\
& =\nabla_{\xi} \varphi_{r}-(-1)^{r}\left(r-\frac{m}{2}\right) \xi \cdot \varphi_{r}
\end{aligned}
$$

and on the other hand, for every $Z \in \Gamma(Q)$,

$$
\nabla_{Z}^{M} \varphi_{r}=\nabla_{Z} \varphi_{r}+\frac{1}{2} \xi \cdot h(Z) \cdot \varphi_{r}
$$

For $r=0$ the identity (9) becomes

$$
\nabla_{\xi}^{M} \varphi_{0}=\nabla_{\xi} \varphi_{0}+\frac{m}{2} \xi \cdot \varphi_{0}
$$

and for the identity (10) we write

$$
\begin{aligned}
\nabla_{Z}^{M} \varphi_{0} & =\nabla_{Z} \varphi_{0}-\frac{1}{2} h(Z) \cdot \xi \cdot \varphi_{0} \\
& =\nabla_{Z} \varphi_{0}-\frac{1}{2} Z \cdot \varphi_{0}
\end{aligned}
$$

for every $Z \in \Gamma(Q)$. So that bringing together (9) and (10) we deduce that the spinor field $\varphi_{0}$ is a $-\frac{1}{2}$-Killing spinor on $M$ if and only if it is a $\left(-\frac{m+1}{2}, 0\right)$-transversal Killing spinor. Now if $\alpha<0$ there exists a $t>0$ such that $\frac{\alpha}{t}=-\frac{m+1}{2}$ so that from Lemma 3.3 there exists a $\mathcal{D}$-homothetic deformation $\bar{g}_{t}$ of the metric $g$ for which the corresponding spinor field ${\overline{\psi_{0}}}^{t}$ is a $\left(-\frac{m+1}{2}, 0\right)$-transversal Killing spinor and hence a $-\frac{1}{2}$-Killing spinor on $\left(M, \bar{g}_{t}\right)$. Furthermore from the argument above the space of $-\frac{1}{2}$-Killing spinors in $\Sigma_{0} M$ exactly coincides with that of $\left(-\frac{m+1}{2}, 0\right)$-transversal Killing spinors in $\Sigma_{0} M$. This proves (i). For $r=m$ the proof is completely analogous.

Notes 4.4 The identities (6) in the proof of Proposition 4.1 actually provide a link between the sign of $\alpha$ and the geometry of $M$. For example if $m$ is odd then the condition $\psi_{0} \neq 0$ (or alternatively $\psi_{m} \neq 0$, which gives the same result) implies from (6) $\operatorname{Scal}_{M}=-2 m-8 \alpha m$. If $\mathrm{Scal}_{M}>0$ then necessarily $\alpha<0$, in particular there is no non-trivial such $(\alpha, 0)$-transversal Killing spinor for some positive $\alpha$ if $\mathrm{Scal}_{M}>0$ and $m$ is odd. This will be illustrated with the Berger spheres in the next section.

Corollary 4.5 Let $\left(M^{2 m+1}, g, \xi\right)$ be a simply-connected complete spin Sasakian manifold carrying a non-zero $(\alpha, \beta)$-transversal Killing spinor $\psi$ for real $\alpha$ and $\beta$. Then we have:

(i) In the case $\alpha=0$ the following holds:

- If $m=1$ then the manifold $M$ is isometric to $\left(\mathbb{S}^{3}\right.$, can $)$, up to $\mathcal{D}$-homothetic deformation of $g$, in the case $\beta \neq 0$ and is diffeomorphic to $\mathbb{R}^{3}$ in the case $\beta=0$.

- If $m>1$ then $\beta=0$, i.e., $\psi$ is a transver sally parallel spinor on $M$. 
(ii) In the case $\alpha \neq 0$ the following holds:

- If $m=1$ then up to $\mathcal{D}$-homothetic deformation of $g$ the manifold $M$ is isometric to $\left(\mathbb{S}^{3}\right.$, can) if $\alpha<0$ and should satisfy $\operatorname{Ric}_{M}=-3 \operatorname{Id}_{T M}+5 \xi^{b} \otimes \xi$ if $\alpha>0$.

- If $m$ is even, $\psi_{0} \neq 0$ and $\alpha<0$ (or $\psi_{m} \neq 0$ and $\alpha>0$ respectively) then $M$ is compact and up to $\mathcal{D}$-homothetic deformation of $g$ it is Einstein-Sasakian.

- If $m \geq 3$ is odd, $\psi_{0}+\psi_{m} \neq 0$ and $\alpha<0$ then $M$ is compact and up to $\mathcal{D}$-homothetic deformation of $g$ it is Einstein-Sasakian or 3-Sasakian.

Proof From Proposition 4.1 we know that $\alpha \beta=0$. In the case where $\alpha=0$ and $\beta \neq 0$, it follows from the identities (6) that $M$ is Einstein if and only if $\beta^{2}=\frac{m+1}{2(2 m-1)}$. Obviously there exists a $t>0$ such that $\frac{\beta^{2}}{t}=\frac{m+1}{2(2 m-1)}$ so that we deduce the existence of a $\mathcal{D}$-homothetic deformation $\bar{g}_{t}$ of $g$ for which $\left(M, \bar{g}_{t}\right)$ is Einstein with positive Ricci curvature. Since $\left(M, \bar{g}_{t}\right)$ is moreover complete (for $(M, g)$ is complete if and only if $\left(M, \bar{g}_{t}\right)$ is) it is compact. One can now adapt an argument $\grave{a}$ la Hijazi to show the non-existence of non-zero basic Killing spinors on $(2 m+1 \geq 5)$-dimensional compact Riemannian flows with a transversal Kähler structure, see e.g. [16, Theorem 5.22]. Therefore, for $m>1$ then necessarily $\beta=0$. Now if $m=1$ and $\beta \neq 0$, we deduce with the fact $\left(M, \bar{g}_{t}\right)$ is of constant curvature, since it is Einstein, that $\left(M^{3}, \bar{g}_{t}\right)$ is isometric to $\left(\mathbb{S}^{3}\right.$, can) [8]. If $m=1$ and $\beta=0$ then using (6) the transversal Ricci curvature vanishes and the manifold $M$ is diffeomorphic to $\mathbb{R}^{3}$ [9]. This shows (i).

Assume now $\alpha \neq 0$. Then from Proposition $4.1 \beta=0$ and if we assume moreover that $\psi_{0} \neq 0$ and $\alpha<0$ (resp. $\psi_{m} \neq 0$ and $(-1)^{m} \alpha>0$ ) then from Proposition 4.3(a) there exists a $\mathcal{D}$-homothetic deformation $\bar{g}_{t}$ of $g$ for which ${\overline{\psi_{0}}}^{t}$ is a $-\frac{1}{2}$-Killing spinor on the Sasakian manifold $\left(M^{2 m+1}, \bar{g}_{t}, \bar{\xi}^{t}\right)$. In particular it is Einstein with scalar curvature $2 m(2 m+1)$ and since $\bar{g}_{t}$ is complete $M$ must actually be compact. In the case where $m$ is odd both conditions are equivalent $(\alpha<0)$. Furthermore if $m \geq 3$ then it follows from C. Bär's classification [4] that $\left(M, \bar{g}_{t}\right)$ should be either Einstein-Sasakian or 3-Sasakian. If $m=1$ the condition $\psi_{0}+$ $\psi_{1} \neq 0$ is fulfilled by hypothesis; if $\alpha<0$ then applying again Proposition 4.3 we obtain a $\mathcal{D}$-homothetic deformation $\bar{g}_{t}$ of $g$ for which $\left(M, \bar{g}_{t}\right)$ carries a non-zero $-\frac{1}{2}$-Killing spinor. Hence similarly one concludes that $\left(M^{3}, \bar{g}_{t}\right)=\left(\mathbb{S}^{3}\right.$, can). If $m=1$ and $\alpha>0$ then from the identities (6) the Ricci curvature is given by $\operatorname{Ric}_{M}=-(4 \alpha+2) \operatorname{Id}_{T M}+4(\alpha+1) \xi^{b} \otimes \xi$, therefore $\left(M^{3}, \bar{g}_{t}\right)$ satisfies $\operatorname{Ric}_{M}=-3 \operatorname{Id}_{T M}+5 \xi^{b} \otimes \xi$ for some $t>0$. This shows (ii) and achieves the proof.

Note 4.6 It also follows from C. Bär's classification [4] that, conversely, if $\left(M^{4 l+1}, g, \xi\right)$ is a complete simply-connected Einstein-Sasakian manifold, then $M$ is spin and carries nontrivial Killing spinors associated to positive and negative real constants. In the case where $m$ is even we therefore obtain examples of such Riemannian flows with non-trivial transversal Killing spinors as soon as e.g. one of those constants can be chosen to be $-\frac{1}{2}$ and the corresponding Killing spinor lies pointwise in $\Sigma_{0} M$. One can obtain such examples when $m \geq 5$ is odd in an analogous way.

\subsection{Example: transversal Killing spinors on the Berger spheres}

For a positive integer $m$ let $M:=\mathbb{S}^{2 m+1}$ be the $(2 m+1)$-dimensional sphere equipped with the round metric $g$ with sectional curvature 1 and its canonical spin structure. It is a Sasakian manifold w.r.t. the vector field $\xi_{x}:=i x$ for every $x \in \mathbb{S}^{2 m+1}$, where $\mathbb{S}^{2 m+1} \subset \mathbb{C}^{m+1}$ and 
$i^{2}=-1$. We shall call the $\mathcal{D}$-homothetic deformations of that Sasakian manifold the Berger spheres. Note that the usual convention is to define a Berger metric on $\mathbb{S}^{2 m+1}$ as $t g_{\mathbb{R} \xi}+g_{\left.\right|_{Q}}$ for some $t>0$.

Let the orientation of $\mathbb{S}^{2 m+1}$ be such that for every positively oriented basis $\left\{e_{1}, \ldots, e_{2 m+1}\right\}$ of $T_{x} \mathbb{S}^{2 m+1}$, the basis $\left\{x, e_{1}, \ldots, e_{2 m+1}\right\}$ is positively oriented in $\mathbb{C}^{m+1}$. Choose $v_{x}:=x$ as unit normal on $\mathbb{S}^{2 m+1}$. Then one can identify $h$ with $J$ (the standard complex structure on $\mathbb{C}^{m+1}$ restricted to $Q$ ).

Proposition 4.7 There exists a 1-dimensional space of $\left(-\frac{m+1}{2}, 0\right)$-(resp. of $\left.\left((-1)^{m} \frac{m+1}{2}, 0\right)-\right)$ transversal Killing spinors on $\mathbb{S}^{2 m+1}$ lying pointwise in $\Sigma_{0} M\left(\right.$ resp. $\left.\Sigma_{m} M\right)$.

Proof It is elementary to show that the space of $-\frac{1}{2}$-Killing spinors on $\mathbb{S}^{2 m+1}$ lying pointwise in $\Sigma_{0} M$ is one-dimensional, as well as the space of $\frac{(-1)^{m}}{2}$-ones in $\Sigma_{m} M$. The result is then a straightforward consequence of Proposition 4.3.

There are no other transversal Killing spinors on the Berger spheres as those that have already been constructed: this is the statement of the following proposition, of which proof is left to the reader.

Proposition 4.8 Let $m \geq 1$ and assume the existence of a non-zero $(\alpha, \beta)$-transversal Killing spinor $\psi$ on $\left(\mathbb{S}^{2 m+1}, g\right)$ for complex $\alpha, \beta$. Then $\alpha \beta=0$ and

(i) If $\alpha=0$ then $m=1, \beta^{2}=1$ and $\psi$ is one of the spinors constructed in Examples 3.8.4.

(ii) If $\alpha \neq 0$ then $\beta=0, \alpha=\epsilon \frac{m+1}{2}$ for some $\epsilon \in\{ \pm 1\}$ and $\psi$ is one of the spinors constructed in Proposition 4.7.

\section{Notes 4.9}

1. There exists in particular no non-zero $\left(\frac{m+1}{2}, 0\right)$-transversal Killing spinor on $\left(\mathbb{S}^{2 m+1}, g\right)$ with $m$ odd, although the space of $\left(-\frac{m+1}{2}, 0\right)$-transversal Killing spinors is 2-dimensional (compare with the case $m$ even). In particular the complex number $\alpha$ cannot be arbitrarily changed into $-\alpha$.

2. Let $M:=\Gamma \backslash \mathbb{S}^{3}$ where $\Gamma$ is a non-trivial finite subgroup of $\mathrm{SU}_{2}$. Remember that, denoting by $\mathrm{SU}(2) \stackrel{\Theta}{\longrightarrow} \mathrm{SO}_{3}$ be the universal covering map of $\mathrm{SO}_{3}$, the group $\Gamma$ is conjugated to a subgroup of one of the following subgroups of $\mathrm{SU}_{2}$ (see e.g. [2, Remark p. 57] or [26, Theorem 2.6.7], where certain subgroups of $\mathrm{SU}_{2}$ are obviously missing): the cyclic group of order $k(k \in \mathbb{N} \backslash\{0\})$ generated by the element $\left(\begin{array}{cc}e^{\frac{2 i \pi}{k}} & 0 \\ 0 & e^{-\frac{2 i \pi}{k}}\end{array}\right) \in \mathrm{SU}_{2}, D_{k}^{*}:=$ $\Theta^{-1}\left(D_{k}^{+}\right)\left(\right.$resp. $T^{*}:=\Theta^{-1}\left(T^{+}\right), O^{*}:=\Theta^{-1}\left(O^{+}\right)$and $\left.I^{*}:=\Theta^{-1}\left(I^{+}\right)\right)$where $D_{k}^{+}($resp. $\mathrm{T}^{+}, \mathrm{O}^{+}$and $\mathrm{I}^{+}$) is the group of orientation-preserving isometries of a regular $k$-gon (resp. tetrahedron, octahedron, and icosahedron). Every such quotient endowed with the metric $g$ induced by the standard metric on $\mathbb{S}^{3}$ is of course again a Sasakian manifold. Moreover it is spin and carries a spin structure for which the space of $\frac{1}{2}$-Killing spinors on $(M, g)$ is 2-dimensional resp. a spin structure for which the space of $-\frac{1}{2}$-Killing spinors on $(M, g)$ is 2-dimensional see [2, Corollary 5.2.5]. Hence there exists for the latter spin structure a 2-dimensional space of $(-1,0)$-transversal Killing spinors. 


\section{Transversal Killing spinors on 3-dimensional flows}

5.1 Integrability conditions for transversal Killing spinors on 3-dimensional flows

In this section we assume that $(M, g, \mathcal{F})$ is a 3-dimensional Riemannian flow. We fix the orientation on $Q$ induced by those of $M$ and $\xi$ (i.e., a basis $\{Z, W\}$ of $Q$ is oriented w.r.t. that orientation if and only if $\{\xi, Z, W\}$ is oriented as local basis of $T M$ ).

A first consequence of the dimension of $M$ being 3 is the existence of an almostHermitian structure $J$ on $Q$ defined in a local positively-oriented orthonormal basis $\left\{e_{1}, e_{2}\right\}$ of $Q$ by the matrix

$$
J:=\left(\begin{array}{cc}
0 & -1 \\
1 & 0
\end{array}\right) .
$$

It is easy to see that $J$ is well-defined, i.e., doesn't depend on the choice of local basis of $Q$ (this follows from the fact that $\mathrm{SO}_{2}$ is Abelain). Furthermore, $J$ is "Kähler" on $Q$, that is $\nabla J=0$ on $M$. Since $h$ is a skew-symmetric tensor, one may write $h$ as

$$
h=b J
$$

for some smooth globally defined function $b: M \longrightarrow \mathbb{R}$. We recall that the complex volume form

$$
\omega_{3}=-\xi \cdot e_{1} \cdot e_{2}
$$

acts as the identity on the spinor bundle $\Sigma M$. Hence one may identify the Clifford action of any 2 -form with that of forms of lower degrees. On the one hand we have

$$
\xi \cdot Z \cdot=J(Z)
$$

for every $Z \in \Gamma(Q)$ and on the other hand we also have

$$
Z \cdot W \cdot=g(J(Z), W) \xi \cdot-g(Z, W) \operatorname{Id}_{\Sigma M}
$$

for all $Z, W \in \Gamma(Q)$. For example, one can identify the Clifford action of $\Omega$ through that of $\xi$ by $\Omega \cdot \varphi=b \xi \cdot \varphi$ for all $\psi \in \Gamma(\Sigma M)$.

In the following proposition and henceforth we denote by $d b_{\left.\right|_{Q}}:=\sum_{k=1}^{2} e_{k}(b) e_{k}$ (orthogonal projection of $\operatorname{grad}(b)$ onto $Q)$.

Proposition 5.1 Let $\left(M^{3}, g, \mathcal{F}\right)$ be a spin Riemannian flow carrying a non-zero $(\alpha, \beta)$ transversal Killing spinor for real $\alpha$ and $\beta$. Then the following holds:

(i) $\alpha=0$ or $\kappa=0$.

(ii) $d \kappa^{\mathrm{b}}\left(e_{1}, e_{2}\right)=2(\xi(b)-4 \alpha \beta)$.

(iii) For every $Z \in \Gamma(Q)$,

$$
\left\{\begin{aligned}
\operatorname{Scal}_{M}=2\left(4 \beta^{2}-b^{2}-4 \alpha b-\operatorname{div}^{M}(\kappa)\right), & \operatorname{Ric}_{M}(\xi)=\left(2 b^{2}-\operatorname{div}^{M}(\kappa)\right) \xi+J\left(2 b \kappa-d b_{\left.\right|_{Q}}\right), \\
\operatorname{Ric}_{M}(Z)= & 2\left(2 \beta^{2}-b^{2}-2 \alpha b\right) Z+(4 \alpha \beta-\xi(b)) J(Z) \\
& +g\left(J\left(2 b \kappa-d b_{\left.\right|_{Q}}\right), Z\right) \xi+\nabla_{Z} \kappa-g(Z, \kappa) \kappa .
\end{aligned}\right.
$$


Proof We keep the notation $\left\{e_{j}\right\}_{1 \leq j \leq 2}$ for a local orthonormal basis of $Q$ and simplify the terms given in Theorem 3.4. On the one hand

$$
\sum_{j=1}^{2} e_{j} \cdot \nabla_{e_{j}}^{M} \kappa \cdot \psi=d \kappa^{b} \cdot \psi+\left(\operatorname{div}^{M}(\kappa)-|\kappa|^{2}\right) \psi-J\left(\nabla_{\xi} \kappa\right) \cdot \psi+b \kappa \cdot \psi .
$$

On the other hand using $\xi\lrcorner d \kappa^{\mathrm{b}}=\nabla_{\xi} \kappa$, one can straightforward prove that

$$
d \kappa^{\mathrm{b}} \cdot \psi=J\left(\nabla_{\xi} \kappa\right) \cdot \psi+d \kappa^{\mathrm{b}}\left(e_{1}, e_{2}\right) \xi \cdot \psi .
$$

We deduce that

$$
\sum_{j=1}^{2} e_{j} \cdot \nabla_{e_{j}}^{M} \kappa \cdot \psi=\left(\operatorname{div}^{M}(\kappa)-|\kappa|^{2}\right) \psi+d \kappa^{b}\left(e_{1}, e_{2}\right) \xi \cdot \psi+b \kappa \cdot \psi
$$

Furthermore, since $h=b J$ and $J$ is $\nabla$-parallel, one has $\nabla_{X} h=X(b) J$ for every $X \in$ $\Gamma(T M)$. In particular, if $Z \in \Gamma(Q)$,

$$
\left\{\begin{array}{l}
\sum_{k=1}^{2} \xi \cdot e_{k} \cdot \nabla_{Z} h\left(e_{k}\right) \cdot \psi=-2 Z(b) \psi \\
\sum_{k=1}^{2} \xi \cdot e_{k} \cdot \nabla_{e_{k}} h(Z) \cdot \psi=g\left(J\left(d b_{\left.\right|_{Q}}\right), Z\right) \xi \cdot \psi-Z(b) \psi
\end{array}\right.
$$

Moreover $\sum_{j, k=1}^{2} e_{j} \cdot e_{k} \cdot \nabla_{e_{j}} h\left(e_{k}\right) \cdot \psi=-2 J\left(d b_{\left.\right|_{Q}}\right) \cdot \psi$ and $\sum_{j=1}^{2} \nabla_{e_{j}} h\left(e_{j}\right)=J\left(d b_{\left.\right|_{Q}}\right)$. In particular

$$
\sum_{j, k=1}^{2} e_{j} \cdot e_{k} \cdot \nabla_{e_{k}} h\left(e_{j}\right) \cdot \psi=0
$$

Last we compute $\sum_{j=1}^{2} e_{j} \cdot \nabla_{\xi} h\left(e_{j}\right) \cdot \psi=2 \xi(b) \xi \cdot \psi$. Now we begin with the proof of the proposition. From Theorem 3.4 we have

$$
\begin{aligned}
\operatorname{Scal}_{M} \psi= & 2\left(4 \beta^{2}-b^{2}-4 \alpha b-\operatorname{div}^{M}(\kappa)\right) \psi \\
& +\left\{4(\xi(b)-4 \alpha \beta)-2 d \kappa^{\mathrm{b}}\left(e_{1}, e_{2}\right)\right\} \xi \cdot \psi+4 \alpha \kappa \cdot \psi .
\end{aligned}
$$

Taking the Hermitian scalar product of this last identity with $\psi$ and identifying the real parts we obtain $\operatorname{Scal}_{M}=2\left\{4 \beta^{2}-b^{2}-4 \alpha b-\operatorname{div}^{M}(\kappa)\right\}$ and for what remains we deduce that $2(\xi(b)-4 \alpha \beta)-d \kappa^{b}\left(e_{1}, e_{2}\right)=0$ and $\alpha \kappa=0$. In particular either $\alpha=0$ or $\kappa=0$. Coming back to the equations involving $\operatorname{Ric}_{M}$, we have on the one hand

$$
\operatorname{Ric}_{M}(\xi) \cdot \psi=\left(2 b^{2}-\operatorname{div}^{M}(\kappa)\right) \xi \cdot \psi+2 b J(\kappa) \cdot \psi-J\left(d b_{\left.\right|_{Q}}\right) \cdot \psi
$$

from which we deduce $\operatorname{Ric}_{M}(\xi)=\left(2 b^{2}-\operatorname{div}^{M}(\kappa)\right) \xi+J\left(2 b \kappa-d b_{\mid Q}\right)$. On the other hand, it also follows from Theorem 3.4 that, for every $Z \in \Gamma(Q)$,

$$
\begin{aligned}
\operatorname{Ric}_{M}(Z) \cdot \psi= & 2\left(2 \beta^{2}-b^{2}-2 \alpha b\right) Z \cdot \psi+(4 \alpha \beta-\xi(b)) J(Z) \cdot \psi \\
+ & \left\{2 b g(J(\kappa), Z)-g\left(J\left(d b_{\left.\right|_{Q}}\right), Z\right)\right\} \xi \cdot \psi+\nabla_{Z} \kappa \cdot \psi \\
& -g(Z, \kappa) \kappa \cdot \psi,
\end{aligned}
$$


from which we deduce that

$$
\begin{aligned}
\operatorname{Ric}_{M}(Z)= & 2\left(2 \beta^{2}-b^{2}-2 \alpha b\right) Z+(4 \alpha \beta-\xi(b)) J(Z) \\
& +g\left(J\left(2 b \kappa-d b_{\left.\right|_{Q}}\right), Z\right) \xi+\nabla_{Z} \kappa-g(Z, \kappa) \kappa .
\end{aligned}
$$

Hence the proof of the proposition is achieved.

It follows from Proposition 5.1 that either $\alpha=0$ or $\kappa=0$. Let us examine the last condition.

Proposition 5.2 Let $\left(M^{3}, g, \mathcal{F}\right)$ be a spin Riemannian flow carrying a non-zero $(\alpha, \beta)$ transversal Killing spinor for real $\alpha$ and $\beta$. Assume that $\kappa=0$.

(i) For every $Z \in \Gamma(Q)$ one has

$$
\left\{\begin{array}{l}
\operatorname{Scal}_{M}=2\left(4 \beta^{2}-b^{2}-4 \alpha b\right), \\
\operatorname{Ric}_{M}(\xi)=2 b^{2} \xi-J\left(d b_{\left.\right|_{Q}}\right), \\
\operatorname{Ric}_{M}(Z)=2\left(2 \beta^{2}-b^{2}-2 \alpha b\right) Z-g\left(J\left(d b_{\left.\right|_{Q}}\right), Z\right) \xi
\end{array}\right.
$$

(ii) One has

$$
\left\{\begin{array}{l}
\nabla_{\xi} d b_{\left.\right|_{Q}}=0, \\
\operatorname{div}^{M}\left(J\left(d b_{\left.\right|_{Q}}\right)\right)=-8 \alpha \beta(3 b+2 \alpha) .
\end{array}\right.
$$

(iii) If furthermore $M$ is compact, then $\alpha \beta=0$ and $\xi(b)=0$.

Proof If $\kappa=0$ then the equations of Proposition 5.1 obviously simplify to the equations in (i). It follows from those equations that $M$ is $\eta$-Einstein if and only if $b$ is constant. Moreover, one may write the Ricci tensor of $M$ in the following way:

$$
\begin{aligned}
\operatorname{Ric}_{M}= & 2\left(2 \beta^{2}-b^{2}-2 \alpha b\right) \operatorname{Id}_{T M}+4\left(b^{2}+\alpha b-\beta^{2}\right) \xi^{b} \otimes \xi \\
& -J\left(d b_{\left.\right|_{Q}}\right)^{b} \otimes \xi-\xi^{b} \otimes J\left(d b_{\left.\right|_{Q}}\right) .
\end{aligned}
$$

Since the divergence of $\xi$ vanishes by the fact that $h$ is skew-symmetric. Using the identity $\operatorname{div}^{M}\left(X^{\mathrm{b}} \otimes Y\right)=\operatorname{div}^{M}(X) Y-\nabla_{X}^{M} Y$ for $X, Y \in \Gamma(T M)$ one obtains, on the one hand, $\operatorname{div}^{M}\left(\xi^{b} \otimes \xi\right)=-\kappa=0$. Similarly, one has

$$
\left\{\begin{array}{l}
\operatorname{div}^{M}\left(\xi^{b} \otimes J\left(d b_{\left.\right|_{Q}}\right)\right)=-\nabla_{\xi} J\left(d b_{\left.\right|_{Q}}\right)+b d b_{\left.\right|_{Q}}, \\
\operatorname{div}^{M}\left(J\left(d b_{\left.\right|_{Q}}\right)^{b} \otimes \xi\right)=\operatorname{div}^{M}\left(J\left(d b_{\left.\right|_{Q}}\right)\right) \xi+b d b_{\left.\right|_{Q}} .
\end{array}\right.
$$

Therefore we can compute the divergence of $\operatorname{Ric}_{M}$ and obtain

$$
\operatorname{div}^{M}\left(\operatorname{Ric}_{M}\right)=2(b+2 \alpha) d b_{\mid Q}+\nabla_{\xi} J\left(d b_{\mid Q}\right)-\left(4 b \xi(b)+\operatorname{div}^{M}\left(J\left(d b_{\left.\right|_{Q}}\right)\right)\right) \xi .
$$

On the other hand we have $d \operatorname{Scal}_{M}=-4(b+2 \alpha) d b_{\left.\right|_{Q}}-4(b+2 \alpha) \xi(b) \xi$. The identity $\operatorname{div}^{M}\left(\operatorname{Ric}_{M}\right)=-\frac{1}{2} d \operatorname{Scal}_{M}$ implies together with the fact that $J$ is parallel w.r.t. the connection $\nabla$,

$$
2(b+2 \alpha) d b_{\left.\right|_{Q}}+J\left(\nabla_{\xi} d b_{\left.\right|_{Q}}\right)-\left(4 b \xi(b)+\operatorname{div}^{M}\left(J\left(d b_{\left.\right|_{Q}}\right)\right)\right) \xi=2(b+2 \alpha)\left(d b_{\left.\right|_{Q}}+\xi(b)\right) \xi,
$$


that is,

$$
\left\{\begin{array}{l}
\nabla_{\xi} d b_{\left.\right|_{Q}}=0, \\
2(3 b+2 \alpha) \xi(b)+\operatorname{div}^{M}\left(J\left(d b_{\left.\right|_{Q}}\right)\right)=0 .
\end{array}\right.
$$

Now remember that from Proposition 5.1 one has $\xi(b)=4 \alpha \beta$, since the mean curvature vanishes. Hence the second equation in (11) may be rewritten under the form $\operatorname{div}^{M}\left(J\left(d b_{\left.\right|_{Q}}\right)\right)=$ $-8 \alpha \beta(3 b+2 \alpha)$. For the rest of the proof assume $M$ to be compact. If $\alpha \beta$ did not vanish, then one would get from Stokes Theorem that

$$
3 \int_{M} b v_{g}+2 \alpha \operatorname{Vol}(M)=0
$$

On the other hand, still following from Stokes Theorem, one would have

$$
-8 \alpha \beta \int_{M} b(3 b+2 \alpha) v_{g}=\int_{M} b \operatorname{div}^{M}\left(J\left(d b_{\mid Q}\right)\right) v_{g}=\int_{M} g\left(d b, J\left(d b_{\mid Q}\right)\right) v_{g}=0,
$$

which would imply

$$
3 \int_{M} b^{2} v_{g}+2 \alpha \int_{M} b v_{g}=0 .
$$

Combining (13) with (12) one would have in particular

$$
\int_{M} b^{2} v_{g}=-\frac{2 \alpha}{3} \int_{M} b v_{g}=\frac{4 \alpha^{2}}{9} \operatorname{Vol}(M)=\frac{\left(\int_{M} b v_{g}\right)^{2}}{\operatorname{Vol}(M)}
$$

i.e., $\frac{1}{\operatorname{Vol}(M)} \int_{M} b^{2} v_{g}=\left(\frac{1}{\operatorname{Vol}(M)} \int_{M} b v_{g}\right)^{2}$. But this is the equality-case in Cauchy-Schwarz inequality, so that $b$ should be constant, which in turn would imply $4 \alpha \beta=\xi(b)=0$ contradiction. Therefore, if $M$ is compact, then $\alpha \beta=0$ in particular $\xi(b)=0$.

5.2 Compact $\eta$-Einstein 3-dimensional minimal flows with transversal Killing spinors

In this section we describe all compact $\eta$-Einstein 3-dimensional minimal flows with transversal Killing spinors for real constants $\alpha$ and $\beta$.

Corollary 5.3 Let $\left(M^{3}, g, \mathcal{F}\right)$ be a Riemannian flow carrying a non-zero $(\alpha, \beta)$-transversal Killing spinor for real $\alpha$ and $\beta$. Assume that $\kappa=0$. Then the following propositions are equivalent:

(i) The Riemannian flow $\left(M^{3}, g\right.$, $\left.\xi\right)$ is $\eta$-Einstein.

(ii) The function $b$ (which is defined by $h=b J$ ) is constant.

(iii) The manifold $M$ is either a local Riemannian product or a Sasakian manifold up to homothety on the metric.

Proof The equivalence of (i) with (ii) is a direct consequence of Proposition 5.2(i). As for the equivalence of (ii) with (iii), one should consider the two cases. The first case is where $b=0$ which gives the vanishing of the O'Neill tensor and with the assumption $\kappa=0$ we deduce that $M$ is locally a Riemannian product. The second case is where $b \neq 0$ which implies that the manifold $\left(M, b^{2} g, \frac{1}{b} \xi\right)$ is Sasakian. 
Proposition 5.4 Let $\left(M^{3}, g, \mathcal{F}\right)$ be a spin compact Riemannian flow carrying a non-zero $(\alpha, \beta)$-transversal Killing spinor. Assume that the flow is minimal, $\eta$-Einstein and that $\alpha, \beta \in$ $\mathbb{R}$. Then $\alpha \beta=0$ and up to homotheties and $\mathcal{D}$-homothetic deformations of the metric $g$ the manifold $M$ is isometric to one of the following:

(i) If $\beta \neq 0: \mathbb{S}^{1} \times \mathbb{S}^{2}, \mathbb{S}^{3}, \mathbb{Z}_{k} \backslash \mathbb{S}^{3}$ for some $k$.

(ii) If $\beta=0: \Gamma \backslash \mathbb{S}^{3}$ for some finite subgroup $\Gamma \subset \mathrm{SU}_{2}$ (if $\left.\alpha<0\right), \Gamma \backslash \widehat{\mathrm{PSL}_{2}(\mathbb{R}) \text { for some }}$ finite cocompact subgroup of $\mathrm{Isom}_{+}\left(\widetilde{\mathrm{PSL}_{2}(\mathbb{R})}\right)$ (if $\alpha>0$ ), a Heisenberg manifold $M_{r}$ (if $\alpha=0$ ) or a Bieberbach manifold $\Gamma \backslash \mathbb{R}^{3}$.

Proof We already know from Proposition 5.2 and Corollary 5.3 that $\alpha \beta=0$ and that $b$ should be constant. Hence for $b=0$, the manifold $M$ is locally a product of two Riemannian manifolds, whereas for $b \neq 0$ it is a Sasakian manifold up to homothety on the metric. We consider the two cases separately:

- Case where $b=0$ : It follows that the universal cover of $M$ is isometric to $\mathbb{R} \times \bar{N}$ where $\bar{N}$ is a simply-connected complete Riemannian surface carrying a $\beta$-Killing spinor. In dimension 2 the only such surfaces are-up to homothety on the metric- $\mathbb{R}^{2}$ (for $\beta=0$ ) and $\mathbb{S}^{2}$ (for $\beta \neq 0$ ), so that $\widetilde{M}$ is isometric to $\mathbb{R}^{3}$ or to $\mathbb{R} \times \mathbb{S}^{2}$ (remember that $\beta$ is assumed to be real).

- In the subcase $\bar{N}=\mathbb{S}^{2}$ the fundamental group of $M$ should be embedded in the product $\operatorname{Isom}_{+}(\mathbb{R}$, can $) \times \operatorname{Isom}_{+}\left(\bar{N}, g_{\bar{N}}\right)$ where $\operatorname{Isom}_{+}$denotes the group of orientationpreserving isometries of the corresponding Riemannian manifold. Since the only orienta tion-preserving isometry subgroup of $\mathrm{SO}_{3}=\operatorname{Isom}_{+}\left(\mathbb{S}^{2}\right)$ acting freely on $\mathbb{S}^{2}$ is the trivial one, we deduce that $\pi_{1}(M)$ is a (discrete) subgroup of $\mathbb{R}=\operatorname{Isom}_{+}(\mathbb{R}$, can), so that $M$ is either isometric to $\mathbb{R} \times \mathbb{S}^{2}$ (if $\pi_{1}(M)=\{\mathrm{Id}\}$ )—which is excluded because of $M$ being assumed to be compact-or to $\mathbb{S}^{1} \times \mathbb{S}^{2}$ (if $\pi_{1}(M) \cong \mathbb{Z}$ ), and in the last situation $\mathbb{S}^{1}$ carries the trivial spin structure.

- In the subcase $\bar{N}=\mathbb{R}^{2}$, i.e. $\beta=0$, the manifold $M$ is Ricci flat hence flat, therefore it is isometric to the quotient $\Gamma \backslash \mathbb{R}^{3}$ where $\Gamma \subset \operatorname{Isom}_{+}\left(\mathbb{R}^{3}\right.$, can $)=\mathbb{R}^{3} \rtimes \mathrm{SO}_{3}$ is a discrete subgroup of orientation-preserving isometries acting freely on $\mathbb{R}^{3}$. In other words, $M$ is one of the Bieberbach manifolds [21].

- Case where $b \neq 0$ : Up to changing $g$ into $b^{2} g$ we may assume that $b=1$ so that $M$ is Sasakian. In that case the assertion follows straightforward from Belgun's uniformization theorem [8] stating that $M$ should be a compact quotient of $\mathbb{S}^{3}, \mathrm{Nil}_{3}$ or $\widetilde{\mathrm{PSL}_{2}(\mathbb{R})}$. This achieves the proof.

Acknowledgements The authors would like to thank the Max-Planck Institute for the sciences and the university of Potsdam as well as the Sonderforschungsbereich 647 "Raum-Zeit-Materie. Analytische und Geometrische Strukturen" of the Deutsche Forschungsgemeinschaft for their support in the preparation of [14] and this paper. It's also a pleasure to thank Bernd Ammann, Christian Bär and Oussama Hijazi for valuable comments.

\section{References}

1. Alexandrov, B., Grantcharov, G., Ivanov, S.: An estimate for the first eigenvalue of the Dirac operator on compact Riemannian spin manifold admitting a parallel one-form. J. Geom. Phys. 28(3-4), 263-270 (1998)

2. Ammann, B.: A variational problem in conformal spin geometry. Habilitation Thesis, Universität Hamburg (2003) 
3. Ammann, B., Bär, C.: The Dirac operator on Nilmanifolds and collapsing circle bundles. Ann. Glob. Anal. Geom. 16, 221-253 (1998)

4. Bär, C.: Real Killing spinors and holonomy. Commun. Math. Phys. 154, 509-521 (1993)

5. Bär, C.: Extrinsic bounds for eigenvalues of the Dirac operator. Ann. Glob. Anal. Geom. 16, 573-596 (1998)

6. Baum, H.: Complete Riemannian manifolds with imaginary Killing spinors. Ann. Glob. Anal. Geom. 7, 205-226 (1989)

7. Baum, H., Friedrich, Th., Grunewald, R., Kath, I.: Twistor and Killing spinors on Riemannian manifolds. Teubner-Texte zur Mathematik, vol. 124. Teubner, Stuttgart (1991)

8. Belgun, F.: On the metric structure of non-Kähler complex surfaces. Math. Ann. 317(1), 1-40 (2000)

9. Blumenthal, R.: Foliated manifolds with flat basic connection. J. Differ. Geom. 16, 401-406 (1981)

10. Boyer, C., Galicki, K.: 3-Sasakian manifolds. In: Surveys in Differential Geometry: Essays on Einstein Manifolds. Surv. Differ. Geom., vol. VI, pp. 123-184. International Press, Boston (1999)

11. Carrière, Y.: Flots Riemanniens. Structure transverse des feuilletages, Toulouse. Astérique 116, 31-52 (1984)

12. Friedrich, Th.: Zur Abhängigkeit des Dirac-Operators von der Spin-Struktur. Coll. Math. J. 48, 57-62 (1984)

13. Friedrich, Th., Kim, E.C.: The Einstein Dirac equations on Riemannian spin manifolds. J. Geom. Phys. 33, 128-172 (2000)

14. Ginoux, N., Habib, G.: A spectral estimate for the Dirac operator on Riemannian flows. Preprint (2007)

15. Habib, G.: Tenseur d'impulsion-énergie et feuilletages. Ph.D. Thesis, Institut Élie Cartan-Université Henri Poincaré, Nancy (2006)

16. Hijazi, O.: Spectral properties of the Dirac operator and geometrical structures. In: Proceedings of the Summer School on Geometric Methods in Quantum Field Theory, Villa de Leyva, Colombia, July 1230, 1999. World Scientific, Singapore (2001)

17. Kirchberg, K.-D.: An estimation for the first eigenvalue of the Dirac operator on closed Kähler manifolds of positive scalar curvature. Ann. Glob. Anal. Geom. 4, 291-325 (1986)

18. Moroianu, A.: Opérateur de Dirac et submersions riemanniennes. Ph.D. Thesis, École Polytechnique (1996)

19. Okumura, M.: Some remarks on space with a certain contact structure. Tôhoku Math. J. (2) 14, 135-145 (1962)

20. O’Neill, B.: The fundamental equations of a submersion. Mich. Math. J. 13, 459-469 (1966)

21. Pfäffle, F.: The Dirac spectrum of Bieberbach manifolds. J. Geom. Phys. 35(4), 367-385 (2000)

22. Reinhart, B.: Foliated manifolds with bundle-like metrics. Ann. Math. 69, 119-132 (1959)

23. Tanno, S.: The topology of contact Riemannian manifolds. Ill. J. Math. 12, 700-717 (1968)

24. Tondeur, Ph.: Foliations on Riemannian Manifolds. Springer, New York (1959)

25. Wang, Mc.K.: Parallel spinors and parallel forms. Ann. Glob. Anal. Geom. 7, 59-68 (1989)

26. Wolf, J.A.: Spaces of Constant Curvature. McGraw-Hill, New York (1967) 